\title{
Finite-Time Output Feedback Controller Based on Observer for the Time-Varying Delayed Systems: A Moore-Penrose Inverse Approach
}

\author{
Cong-Trang Nguyen and Yao-Wen Tsai \\ Mechanical and Automation Engineering, Da-Yeh University, Changhua 51591, Taiwan \\ Correspondence should be addressed to Yao-Wen Tsai; ywtsai@mail.dyu.edu.tw
}

Received 30 December 2016; Revised 10 April 2017; Accepted 12 April 2017; Published 24 May 2017

Academic Editor: R. Aguilar-López

Copyright (C) 2017 Cong-Trang Nguyen and Yao-Wen Tsai. This is an open access article distributed under the Creative Commons Attribution License, which permits unrestricted use, distribution, and reproduction in any medium, provided the original work is properly cited.

\begin{abstract}
This study proposes a novel variable structure control (VSC) for the mismatched uncertain systems with unknown time-varying delay. The novel VSC includes the finite-time convergence sliding mode, invariance property, asymptotic stability, and measured output only. A necessary and sufficient condition guaranteeing the existence of sliding surface is given. A novel lemma is established to deal with the control design problem for a wider class of time-delay systems. A suitable reduced-order observer (ROO) is constructed to estimate unmeasured state variables of the systems. A novel finite-time output feedback controller (FTOFC) is investigated, which is based on the ROO tool and the Moore-Penrose inverse technique. Moreover, with the help of this lemma and the proposed FTOFC, restrictions on most existing works are also eliminated. In addition, an asymptotic stability analysis is implemented by means of the feasibility of the linear matrix inequalities (LMIs) and given desirable sliding mode dynamics. Finally, a MATLAB simulation result on a numerical example is performed to show the effectiveness and advantage of the proposed method.
\end{abstract}

\section{Introduction}

Time-delay systems are one of the main topics of control systems which have been successfully applied by the variable structure control (VSC) theory $[1,2]$. It commonly leads to an instability and/or reduces the system performance in the closed-loop system [3]; hence, the stability of the time-delay uncertain system has been attracting the interest of a large number of quality papers published in the most recently internationally renowned journals [4-8] and the related references therein. Thanks are due to some distinguished features of the VSC such as finite-time convergence, fast dynamic response, good robustness, exogenous perturbations rejection ability, and its insensitivity to parameter variations. The VSC theory has been effectively applied to a wide variety of practical time-delay systems such as hydraulic/pneumatic, data transmission, satellite systems, robotic manipulator, chemical processes, communication, and network system [912].

Based on the published works above, the VSC design largely falls into two categories. The first category is that all unavailable state variables are estimated and called full-order observer (FOO) or a part of the state variables and called a reduced-order observer (ROO), such that error trajectory reached the sliding surface of the error dynamic and the estimated variables tend to the actual variables of systems. The second category is that a control signal of systems is constructed via measured output, called output feedback controller (OFC), such that the state trajectories of systems move onto the sliding surface.

In order to estimate unmeasured states of a plant, there are several FOOs/ROOs that are successfully designed by [13-17]. In [14], the FOO was established for uncertain singleinput/single-output (SISO) and multiple-input/multipleoutput (MIMO) systems which satisfied the matching condition with time-delay. The design parameters of time-delay observer were chosen by using the Lyapunov-Krasovskii Vfunctional method. Based on the generalized matrix inverse concept, the work [15] extended the FOO results of [14] from the matched uncertain systems to mismatched uncertain systems with a time-delay. Nevertheless, the time-delay 
error convergence of asymptotic observer is uniformly ultimately bounded. In contrast to the FOO, the ROO estimates only those states that are not directly measured. An asymptotic observer in a lower dimension was studied in $[13,16]$ for linear time-delay systems by utilizing LMI technique and linear matrix equality formulation. This LMI technique [18] has some benefits over traditional approach methods; that is, LMI problems can be easily determined and efficiently solved by using the LMI Toolbox [19] in MATLAB software. However, all of these techniques have a common disadvantage to providing an asymptotic stability of estimation error in infinite time. For the purpose of control design, the finite-time convergence is one of the most essential and challenging problems. It requires fundamentally that a control system is stable in the sense of Lyapunov and its trajectories tend to zero in finite time. It was demonstrated in [17] that the finite-time convergence of FOO was constructed for the time-varying delay uncertain nonlinear systems under Lipschitz conditions. In brief, the main key for observer progress is a finite-time convergence of estimation error such that observer is invariant to the system uncertainties and/or disturbances in finite time. However, in most existing FOO/ROO works, the finite-time convergence could not be guaranteed simultaneously with the invariance property for mismatched uncertain systems with a time-delay. Further, there are presently only few results in which the time-delay does not need to have prior knowledge in the observer [20-22].

In recent years, the problem of designing a controller for the uncertain system with time-varying delay has achieved a great deal of results [23-27]. Among them, the controller was established in [24] for the matched uncertain SISO/MIMO system with time-varying state-delay and additive disturbances by using an LMI approach. Also, based on benefits of the LMI technique, the controller was designed in [25] for linear time-delay systems with mismatched perturbations. However, the norm of external disturbance is assumed to be bounded a known positive constant. A novel concept, named "a subordinated reachability of the sliding motion," was introduced in [27] for a class mismatched uncertain of the stochastic system with time-varying delay. In [26], by means of a Takagi-Sugeno (T-S) fuzzy modelling approach, the sliding mode control problem was investigated for mismatch uncertain time-delay systems. For a class of uncertain stochastic delay systems in [23], a state feedback controller was developed by using integral sliding mode control approach. However, an asymptotic stability is not ensured the finite-time convergence. Furthermore, these works assumed that all the system states were accessible to the control law. In many practical systems, the state variables are not measured directly or the number of measuring devices is limited. Hence, the works of OFC based on FOOs/ROOs were investigated by many authors $[4-8,28]$. In [28], the OFC was represented for Itô stochastic time-delay systems by utilizing the FOO tool and measured output. But the obtained results could not ensure invariance to the matched uncertainties in sliding mode. The work [6] established OFC which was assumed to satisfy the norm of unmeasured states with known nonnegative constant value. This constant value is not easily achieved in practice. The FOO-based OFC was explored in [7] for uncertain time-delay systems with Markovian jump parameters. In [8], the OFC was designed for uncertain time-delay systems where stochastic perturbations must satisfy stringent Lipschitz condition. Recently, the study [4] was conducted to design OFC based on FOO for nonlinear Markovian jump systems with partly unknown transition probabilities. In [5], the OFC was proposed, which assumed that the norm of states and norm of observer error have to be bounded output signal, for a class of uncertain neural systems with unmeasured states. It should be pointed out that the recent works [4-7] have some serious limitations, where it is required that the exogenous disturbances must be bounded by a known function of the outputs. Moreover, all published works have represented the OFC based on FOO, which increases the computation of burden due to the associated closed-loop systems.

The analysis as mentioned above and the significant limitations of published works have motivated the output variables studies only. It would be worthwhile to design a finite-time output feedback controller (FTOFC) for mismatched uncertain systems with unknown time-delay. The FTOFC will be based on the ROO tool and the MoorePenrose inverse technique in which the above restrictions are relaxed. Hence, a novel VSC approach should be investigated for the mismatched uncertain systems with unknown timevarying delay and external disturbances input. The novel VSC includes the finite-time convergence sliding mode, invariance property, asymptotic stability, eliminated limitations, and measured output only. In this paper, we attempt to develop a novel FTOFC with four main tasks. The first is concerned with a necessary and sufficient condition guaranteeing the existence of sliding surface. The second consists of the construction of a suitable ROO, which estimates unmeasured variables. This ROO ensures that the conservatism is reduced, and the robustness is enhanced in comparison with FOO. The third involves a novel lemma that is established to handle an unknown error of the observer error dynamics in the control design problem. The last one comprises a novel FTOFC, which is designed based on the ROO tool and the Moore-Penrose inverse technique to stabilize the mismatched uncertain systems with unknown time-delay. Thus, the novel method does not need the availability of the state variables; besides, constructing LMI condition to guarantee the timedelay systems with mismatched uncertainties in sliding mode is asymptotically stable. Finally, a numerical example is given to prove the effectiveness of the proposed theoretical results.

The configuration of this paper is organized as follows. The problem statement, preliminaries, and some useful lemmas are introduced in Section 2. Section 3 presents the sliding surface design and regular form of the system. Section 4 shows the important achievements of the paper, which show how to establish the FTOFC based on the ROO tool and the novel lemma for the mismatched uncertain systems with unknown time-varying delay. Section 5 verifies the effectiveness of the proposed design method through a numerical example. Finally, some concluding remarks are epitomized in Section 6. 
Notation. Throughout this paper, $R^{n}$ symbolizes the $n$ dimensional Euclidean space, and $R^{n \times m}$ denotes the set of all $n \times m$ real matrices. For matrix $A$, the notation $A>0$ means that the matrix $A$ is a positive definite matrix. $I$ and 0 represent the identity matrix and a zero matrix, respectively. The superscript " $T$ " shows the transpose. $M^{\perp}$ denotes an orthogonal complement of $M$ (i.e., $M^{\perp T} M=0$ ). Finally, the notation $\|\cdot\|$ stands for the Euclidean norm of a vector and the induced spectral norm of a matrix.

\section{Problem Statement and Preliminaries}

Consider a general mismatched uncertain time-delay system whose dynamics are described by the following equations:

$$
\begin{aligned}
\dot{x}(t)= & {[A+\Delta A(t)] x(t) } \\
& +\left[A_{d}+\Delta A_{d}(t)\right] x(t-d(t)) \\
& +B[u(t)+\xi(x(t), x(t-d(t)), t)], \\
y(t)= & C x(t), \\
x(t)= & \phi(t), \text { for }-\bar{d} \leq t<0,
\end{aligned}
$$

where $x(t) \in R^{n}$ is the system continuous-time state variables, $u(t) \in R^{m}$ represents the control input of the plant, and $y(t) \epsilon$ $R^{p}$ is the measured output. The function $d(t)$ is the timevarying delay which is assumed to be unknown, nonnegative, and bounded in $\mathfrak{R}^{+}$; that is, $\bar{d}:=\sup _{t \in \mathfrak{R}^{+}}\{d(t)\}<\infty$. The symbol $\phi(t)$ represents differential vector-valued initial function on $[-\bar{d}, 0]$. The constant matrices $A, A_{d}, B, C, D$, and $E$ are nonunique constant matrices with appropriate dimensions. The matrices $\Delta A(t)$ and $\Delta A_{d}(t)$ represent the structure parameter mismatched uncertainties in the state matrix and the delayed state matrix, respectively. The term $\xi(x(t), x(t-d(t)), t)$ describes the influence of exogenous disturbance on the plant.

To proceed with the design of the observer and control scheme for the uncertain time-delay systems (1), the following standard assumptions are essential for our work.

Assumption 1. $m \leq p<n$; that is, the number of inputs is smaller than or equal to the number of output channels. The input matrices $B$ and $C$ have full rank, and $\operatorname{rank}(C B)=m$.

Assumption 2. The pair $\left(A+A_{d}, B\right)$ is completely controllable, and the pair $\left(A+A_{d}, C\right)$ is completely observable.

Assumption 3. The mismatched uncertainties $\Delta A(t)$ and $\Delta A_{d}(t)$ are norm bounded; that is,

$$
\begin{aligned}
& {\left[\Delta A(t) \quad \Delta A_{d}(t)\right]} \\
& =D\left[\Sigma(x(t), x(t-d(t)), t) \quad \Sigma_{d}(x(t), x(t-d(t)), t)\right] E,
\end{aligned}
$$

where $\Sigma(x(t), x(t-d(t)), t)$ and $\Sigma_{d}(x(t), x(t-d(t)), t)$ are unknown matrix function satisfying $\|\Sigma(x(t), x(t-d(t)), t)\| \leq$ 1 and $\left\|\Sigma_{d}(x(t), x(t-d(t)), t)\right\| \leq 1$ for all $t \geq 0$, respectively.
Assumption 4. $\xi(x(t), x(t-d(t)), t)$ is an unknown disturbance which satisfies $\|\xi(x(t), x(t-d(t)), t)\| \leq k_{\xi}+k_{m}(\|x(t)\|+$ $\|x(t-d(t))\|)$, where $k_{\xi}$ and $k_{m}$ are known nonnegative constants.

Remark 5. Assumptions 1, 2, and 3 are standard assumptions for time-delay systems which can be found in most existing literatures. For Assumption 4, in recent studies [4-7], it is required in this technical note that the exogenous disturbances must be bounded by a known function of the outputs. In practical cases, these conditions are often difficult to meet. For our method, the external disturbances must satisfy an unknown function of the state and delayed state variables. Thus, the condition in Assumption 4 is an extension of the condition used in these studies.

For further analysis, some following standard lemmas will be needed as follows that are useful for the development of theorems and stability of the system dynamics in sliding mode.

Lemma 6 (see [29]). Let $R_{1}, R_{2}$, and $\Sigma(t)$ be real matrices of suitable dimension with $\Sigma^{T} \Sigma \leq I$; then, for any scalar $\varphi>0$, the following matrix inequality holds:

$$
R_{1} \Sigma(t) R_{2}+R_{2}^{T} \Sigma^{T}(t) R_{1}^{T} \leq \varphi^{-1} R_{1} R_{1}^{T}+\varphi R_{2}^{T} R_{2} .
$$

Lemma 7 (see [30]). For two vectors $x, y$ of $R^{n}$ and a positive definite matrix $N \in R^{n \times n}$, the following inequality holds:

$$
x^{T} N y+y^{T} N x \leq v^{-1} x^{T} N x+v y^{T} N y,
$$

for all $v>0$

Lemma 8 (see [18]). For a given matrix $\Delta=\left[\begin{array}{ll}\Delta_{11} & \Delta_{12} \\ \Delta_{12}^{T} & \Delta_{22}\end{array}\right]$ with $\Delta_{11}^{T}=\Delta_{11}$ and $\Delta_{22}^{T}=\Delta_{22}$, then the following conditions are equivalent:

$$
\begin{aligned}
\text { (i) } & \Delta<0, \\
\text { (ii) } & \Delta_{11}<0, \\
& \Delta_{22}-\Delta_{12}^{T} \Delta_{11}^{-1} \Delta_{12}<0, \\
\text { (iii) } & \Delta_{22}<0, \\
& \Delta_{11}-\Delta_{12}^{T} \Delta_{22}^{-1} \Delta_{12}<0 .
\end{aligned}
$$

\section{Sliding Surface Design and Regular Form of the System}

In this section, we present a procedure for the design of a sliding surface and deriving existence condition of the sliding matrix using only the output variable. After the sliding surface design is completed the next step is to get a regular form of the original system (1) such that we prepare a controller design for system (1).

First, let us define the sliding function as

$$
\sigma(t)=F y(t)=F C x(t)=S x(t)
$$


then the sliding surface, which is defined by $\sigma(t)=0$ with $F \in$ $R^{m \times p}$ is a constant matrix, and $S \in R^{m \times n}$ is a sliding matrix. It follows from (6), one can see that there are only output variables used. According to the existing works $[31,32]$, the following properties for the sliding surface parameter matrix $S$ should be satisfied.

Property 1. The matrix $(S B)$ is nonsingular.

Property 2. The sliding mode dynamics restricted to sliding surface $\sigma(t)=F y(t)=S x(t)=0$ are asymptotically stable and are completely invariant to any uncertainties and/or disturbances satisfying Assumptions 3 and 4.

Property 3. There exists a matrix $F$ such that $S=F C$.

The purpose is to design the FTOFC for system (1); we now consider the results of [31] for the regular form. Let us define by $\Gamma n \times n$ symmetric matrix satisfying

$$
\Gamma= \begin{cases}I, & \text { if } B^{\perp T} D=0 \\ I-E^{g} E, & \text { if } B^{\perp T} D \neq 0,\end{cases}
$$

where $B^{\perp}$ is an orthogonal complement of the matrix $B$ and $E^{g}$ is the Moore-Penrose inverse of the matrix $E$.

Remark 9. We have matching condition where the parameter uncertainties $\Delta A(t)$ and $\Delta A_{d}(t)$ satisfy the term $B^{\perp T} D=0$; that is, the matrix $\Gamma=I$. Otherwise, we have the mismatching condition where the uncertain terms $\Delta A(t)$ and $\Delta A_{d}(t)$ gratify the term $B^{\perp T} D \neq 0$; that is, the matrix $\Gamma=I-E^{g} E$.

Consider the two constraints of the following LMIs:

$$
\begin{array}{r}
\Gamma X \Gamma+B Y B^{T}>0, \\
B^{\perp T}\left(A Г X \Gamma+\Gamma X \Gamma A^{T}\right) B^{\perp}<0,
\end{array}
$$

where $X$ and $Y$ are symmetric matrices.

In this case, the sliding matrix (6) can be parameterized as

$$
S=F C=N B^{T} P^{-1}
$$

where $N$ is any $m \times m$ nonsingular matrix and $P=\Gamma X \Gamma+$ $B Y B^{T}$. The choice of the matrix $F$ and existence of matrix $S$ will be presented in Theorem 11.

Remark 10. According to the existing studies [31, 33, 34], there exists a sliding matrix $S$ guaranteeing Properties 1-3 if and only if there exists a solution pair $(X, Y)$ satisfying the LMIs (8). Then the sliding surface can be parameterized as (9). Additionally, it is easy to show that the LMIs (8) can be solved by using LMI Toolbox [19] in MATLAB software.

To complete the regular form description of system (1), a following transformation matrix is denoted as

$$
T=\left[\begin{array}{c}
B^{\perp T} \\
N B^{T} P^{-1}
\end{array}\right],
$$

and assume that $(S B)$ is nonsingular; then the inverse of $T$ has the form

$$
T^{-1}=\left[P B^{\perp}\left(B^{\perp T} P B^{\perp}\right)^{-1} B(S B)^{-1}\right] .
$$

Now, we describe a system state and the delayed state transformation as

$$
\left[\begin{array}{l}
z(t) \\
\sigma(t)
\end{array}\right]=T x(t)
$$

$$
\left[\begin{array}{l}
z(t-d(t)) \\
\sigma(t-d(t))
\end{array}\right]=T x(t-d(t)),
$$

where the variables $z(t) \in R^{n-m}$ and $z(t-d(t)) \in R^{n-m}$ are unmeasurable, whereas the sliding variables $\sigma(t) \in R^{m}$ and $\sigma(t-d(t)) \in R^{m}$ are measurable.

Computing the derivative of (12) with respect to time, then the original system (1) is equivalent to the following regular form:

$$
\begin{aligned}
& {\left[\begin{array}{l}
\dot{z}(t) \\
\dot{\sigma}(t)
\end{array}\right]} \\
& =\left[\begin{array}{ll}
A_{11}+\Delta A_{11} & A_{12}+\Delta A_{12} \\
A_{21}+\Delta A_{21} & A_{22}+\Delta A_{22}
\end{array}\right]\left[\begin{array}{l}
z(t) \\
\sigma(t)
\end{array}\right] \\
& +\left[\begin{array}{cc}
A_{11 d}+\Delta A_{11 d} & A_{12 d}+\Delta A_{12 d} \\
A_{21 d}+\Delta A_{21 d} & A_{22 d}+\Delta A_{22 d}
\end{array}\right]\left[\begin{array}{l}
z(t-d(t)) \\
\sigma(t-d(t))
\end{array}\right] \\
& +\left[\begin{array}{c}
0 \\
S B
\end{array}\right] u(t)+\left[\begin{array}{c}
0 \\
S B
\end{array}\right] \xi(x(t), x(t-d(t)), t),
\end{aligned}
$$

where

$$
\begin{aligned}
& A_{11}+\Delta A_{11}=B^{\perp T}(A+D \Sigma E) P B^{\perp}\left(B^{\perp T} P B^{\perp}\right)^{-1}, \\
& A_{12}+\Delta A_{12}=B^{\perp T}(A+D \Sigma E) B(S B)^{-1}, \\
& A_{21}+\Delta A_{21} \\
& =N B^{T} P^{-1}(A+D \Sigma E) P B^{\perp}\left(B^{\perp T} P B^{\perp}\right)^{-1}, \\
& A_{22}+\Delta A_{22}=N B^{T} P^{-1}(A+D \Sigma E) B(S B)^{-1}, \\
& A_{11 d}+\Delta A_{11 d} \\
& \quad=B^{\perp T}\left(A_{d}+D \Sigma_{d} E\right) P B^{\perp}\left(B^{\perp T} P B^{\perp}\right)^{-1}, \\
& A_{12 d}+\Delta A_{12 d}=B^{\perp T}\left(A_{d}+D \Sigma_{d} E\right) B(S B)^{-1},
\end{aligned}
$$




$$
\begin{aligned}
& A_{21 d}+\Delta A_{21 d} \\
& \quad=N B^{T} P^{-1}\left(A_{d}+D \Sigma_{d} E\right) P B^{\perp}\left(B^{\perp T} P B^{\perp}\right)^{-1}, \\
& A_{22 d}+\Delta A_{22 d}=N B^{T} P^{-1}\left(A_{d}+D \Sigma_{d} E\right) B(S B)^{-1}, \\
& z(t)=B^{\perp T} x(t), \\
& z(t-d(t))=B^{\perp T} x(t-d(t)), \\
& \sigma(t)=S x(t), \\
& \sigma(t-d(t))=S x(t-d(t)) .
\end{aligned}
$$

From (13), it can be rewritten as

$$
\begin{aligned}
\dot{z}(t)= & {\left[A_{11}+\Delta A_{11}\right] z(t) } \\
& +\left[A_{11 d}+\Delta A_{11 d}\right] z(t-d(t)) \\
& +\left[A_{12}+\Delta A_{12}\right] \sigma(t) \\
& +\left[A_{12 d}+\Delta A_{12 d}\right] \sigma(t-d(t)), \\
\dot{\sigma}(t)= & {\left[A_{21}+\Delta A_{21}\right] z(t) } \\
& +\left[A_{21 d}+\Delta A_{21 d}\right] z(t-d(t)) \\
& +\left[A_{22}+\Delta A_{22}\right] \sigma(t) \\
& +\left[A_{22 d}+\Delta A_{22 d}\right] \sigma(t-d(t)) \\
& +(S B)[u(t)+\xi(x(t), x(t-d(t)), t)],
\end{aligned}
$$

where $z(t)=\phi_{z}(t)=B^{\perp T} \phi(t), \sigma(t)=\phi_{\sigma}(t)=N B^{T} P^{-1} \phi(t)$ with $t \in[-\bar{d}, 0]$

Now, existence condition of the sliding matrix in terms of LMIs using only the output variable is shown in Theorem 11.

Theorem 11. Consider the time-varying delay systems (1) with mismatched uncertainties. Assume that Assumptions 1-4 hold. Let $\Pi=B^{\perp T} P A_{d}^{T} B^{\perp} B^{\perp T} A_{d} P B^{\perp}\left(B^{\perp T} P B^{\perp}\right)^{-1}+B^{\perp T} P B^{\perp}+$ $B^{\perp T}\left(P A^{T}+A P\right) B^{\perp}$. Then there exists a sliding matrix, $S$, such that Properties 1-3 hold if and only if the following LMI has a solution pair $(P, F)$ for any constants $k_{1}>0, k_{2}>0$.

$$
P>0
$$

$$
\begin{aligned}
& {\left[\begin{array}{ccc}
\Pi & B^{\perp T} P B^{\perp} B^{\perp T} D & B^{\perp T} P B^{\perp} B^{\perp T} D \\
D^{T} B^{\perp} B^{\perp T} P B^{\perp} & -k_{1} I & 0 \\
D^{T} B^{\perp} B^{\perp T} P B^{\perp} & 0 & -k_{2} I
\end{array}\right]} \\
& \quad<0 \\
& N B^{T}=F C P .
\end{aligned}
$$

\section{Proof of Theorem 11.}

Necessity. It follows that the first equation of system (15) can be acknowledged as the following sliding mode dynamics of the overall closed-loop systems:

$$
\begin{aligned}
\dot{z}(t)= & {\left[A_{11}+\bar{D} \Sigma \bar{E}\right] z(t) } \\
& +\left[A_{11 d}+\bar{D} \Sigma_{d} \bar{E}\right] z(t-d(t)),
\end{aligned}
$$

where $\bar{D}=B^{\perp T} D$ and $\bar{E}=E P B^{\perp}\left(B^{\perp T} P B^{\perp}\right)^{-1}$.

To determine an existence condition of the sliding matrix, Property 3 holds. We select the Lyapunov function candidate of the form $V(t)=z^{T}(t) H z(t)$, where $H$ is positive matrix. Then, calculating the time derivative of $V(t)$ along the state trajectories of system (18), it can be found that

$$
\begin{aligned}
\dot{V}(t)= & z^{T}(t)\left[A_{11}^{T} H+H A_{11}+\bar{E}^{T} \Sigma^{T} \bar{D}^{T} H+H \bar{D} \Sigma \bar{E}\right] \\
\cdot & z(t)+z^{T}(t-d(t)) \bar{E}^{T} \Sigma_{d}^{T} \bar{D}^{T} H z(t) \\
+ & z^{T} H \bar{D} \Sigma_{d} \bar{E} z(t-d(t))+z^{T}(t) \\
& \cdot H A_{11 d} z(t-d(t))+z^{T}(t-d(t)) \\
& \cdot A_{11 d}^{T} H z(t) .
\end{aligned}
$$

Now, we are going to prove $\dot{V}(t)<0$. By using Lemma 6, it follows from (19) that

$$
\begin{aligned}
\dot{V}(t) \leq & z^{T}(t) \\
& \cdot\left[A_{11}^{T} H+H A_{11}+\varphi^{-1} H \bar{D} \bar{D}^{T} H+\varphi \bar{E}^{T} \bar{E}\right] \\
\cdot & z(t)+\varphi_{1 d}^{-1} z^{T}(t) H \bar{D} \bar{D}^{T} H z(t) \\
+ & \varphi_{1 d} z^{T}(t-d(t)) \bar{E}^{T} \bar{E} z(t-d(t))+z^{T}(t) \\
& \cdot H A_{11 d} z(t-d(t))+z^{T}(t-d(t)) \\
& \cdot A_{11 d}^{T} H z(t) .
\end{aligned}
$$

By virtue of Lemma 7, inequality (20) is equivalent to

$$
\begin{gathered}
\dot{V}(t) \leq z^{T}(t)\left[A_{11}^{T} H+H A_{11}+\varphi^{-1} H \bar{D} \bar{D}^{T} H+\varphi \bar{E}^{T} \bar{E}\right. \\
\left.+\varphi_{2 d}^{-1} H+\varphi_{1 d}^{-1} H \bar{D} \bar{D}^{T} H\right] z(t)+z^{T}(t-d(t)) \\
\cdot\left[\varphi_{1 d} \bar{E}^{T} \bar{E}+\varphi_{2 d} A_{11 d}^{T} H A_{11 d}\right] z(t-d(t)) .
\end{gathered}
$$

According to Assumption 3, $E$ is a free-choice matrix. So, we can easily select matrix $E$ such that the matrix $E^{T} E$ is semipositive definite. Then, from Lemma 3 of [35], the following is true:

$$
\begin{gathered}
z^{T}(t-d(t)) A_{11 d}^{T} H A_{11 d} z(t-d(t)) \\
\leq \mu_{1} z^{T}(t) A_{11 d}^{T} H A_{11 d} z(t)
\end{gathered}
$$


for some $\mu_{1}>1$, which implies that

$$
z^{T}(t-d(t)) \bar{E}^{T} \bar{E} z(t-d(t)) \leq \mu_{2} z^{T}(t) \bar{E}^{T} \bar{E} z(t),
$$

where the scalar $\mu_{2}>1$. Thus, from (21), (22), and (23), we achieve

$$
\begin{aligned}
& \dot{V}(t) \leq z^{T}(t)\left[A_{11}^{T} H+H A_{11}+\left(\varphi+\mu_{2} \varphi_{1 d}\right) \bar{E}^{T} \bar{E}\right. \\
& +\varphi^{-1} H \bar{D} \bar{D}^{T} H+\varphi_{2 d}^{-1} H+\mu_{1} \varphi_{2 d} A_{11 d}^{T} H A_{11 d} \\
& \left.+\varphi_{1 d}^{-1} H \bar{D} \bar{D}^{T} H\right] z(t) .
\end{aligned}
$$

To get $\dot{V}(t)<0$, applying Lemma 8 to the above inequality yields

$$
\left[\begin{array}{cccc}
\Pi & H \bar{D} & H \bar{D} & \bar{E}^{T} \\
\bar{D}^{T} H & -k_{1} I & 0 & 0 \\
\bar{D}^{T} H & 0 & -k_{2} I & 0 \\
\bar{E} & 0 & 0 & -k_{3} I
\end{array}\right]<0
$$

where $H \in R^{(n-m) \times(n-m)}$ is any positive matrix, $\Pi=A_{11}^{T} H+$ $H A_{11}+\mu_{1} \varphi_{2 d} A_{11 d}^{T} H A_{11 d}+\varphi_{2 d}^{-1} H$, and the scalars $k_{1}=\varphi^{-1}>$ $0, k_{2}=\varphi_{1 d}^{-1}>0$, and $k_{3}=\left(\varphi+\mu_{2} \varphi_{1 d}\right)>0$.

Now, assume that if the term $B^{\perp T} D \neq 0$, then the uncertainties $\Delta A(t)$ and $\Delta A_{d}(t)$ will not satisfy the matching condition, and constraint (8) is feasible. It can be easily shown

$$
\begin{aligned}
\Gamma & =I-E^{\mathfrak{g}} E, \\
P & =\Gamma X \Gamma+B Y B^{T}=\left(I-E^{\mathfrak{g}} E\right) X\left(I-E^{\mathfrak{g}} E\right)+B Y B^{T} \\
& >0 .
\end{aligned}
$$

And so, we get

$$
\begin{aligned}
B^{\perp T} P B^{\perp} & =B^{\perp T}\left(I-E^{\mathcal{g}} E\right) X\left(I-E^{\mathcal{g}} E\right) B^{\perp}>0, \\
E P B^{\perp} & =E\left[\left(I-E^{g} E\right) X\left(I-E^{\mathcal{g}} E\right)+B Y B^{T}\right] B^{\perp} \\
& =0 .
\end{aligned}
$$

Thus, we get $\bar{E}=E P B^{\perp}\left(B^{\perp T} P B^{\perp}\right)^{-1}=0$ and select $H=$ $\left(B^{\perp T} P B^{\perp}\right)>0$. The LMI (25) can be rewritten as

$$
\left[\begin{array}{ccc}
\Pi & B^{\perp T} P B^{\perp} \bar{D} & B^{\perp T} P B^{\perp} \bar{D} \\
\bar{D}^{T} B^{\perp T} P B^{\perp} & -k_{1} I & 0 \\
\bar{D}^{T} B^{\perp T} P B^{\perp} & 0 & -k_{2} I
\end{array}\right]<0,
$$

where $\Pi=B^{\perp T} P A^{T} B^{\perp}+B^{\perp T} A P B^{\perp}+$ $\mu_{1} \varphi_{2 d} B^{\perp T} P A_{d}^{T} B^{\perp} B^{\perp T} A_{d} P B^{\perp}\left(B^{\perp T} P B^{\perp}\right)^{-1}+\varphi_{2 d}^{-1}\left(B^{\perp T} P B^{\perp}\right)$, because of $P=\Gamma X \Gamma+B Y B^{T}=\left(I-E^{g} E\right) X\left(I-E^{g} E\right)+B Y B^{T}>0$, as (26) implies that $P$ satisfies the LMI (28).

Sufficiency. Assume that the LMI (17) is feasible, and let sliding matrix, $S$, be $S=F C=N B^{T} P^{-1}$. Clearly, the matrix S satisfies Properties 1 and 2. According to [32], we can see that the reduced-order sliding mode dynamic (18) is asymptotically stable in sliding mode $\sigma(t)=F C x(t)=$ $N B^{T} P^{-1} x(t)=0$; that is, $S=F C=N B^{T} P^{-1}$ satisfies Property 3 . The proof is completed.

Remark 12. When a sliding mode is operated, the first equation of the overall closed-loop system (15) is asymptotically stable by means of the feasibility of LMI (25). This will reduce conservatism in the computing process and ensure robustness against the matched uncertainty and the external perturbation of the time-varying delay system. Besides, the novel existence condition of sliding mode is provided by the LMI (25) with regard to the sliding function (6), which can be easily performed by using LMI Toolbox [19] in MATLAB software.

\section{Design of the Finite-Time Output Feedback Controller Based on ROO}

4.1. Sliding Control Law Construction. In this section, the main results will be shown. The first suitable ROO is established to generate the estimate of unmeasured states of the system. A control law will then be determined by using these estimated variables and the system outputs such that reachability condition

$$
\sigma^{T}(t) \dot{\sigma}(t) \leq-\alpha\|\sigma(t)\|
$$

can be met for some positive scalars $\alpha$, where $\sigma(t)=F y(t)$ is the sliding function. If condition (29) is gratified by some control, then system (1) can be driven from any initial state to reach the sliding surface in finite time and remain there in subsequent time.

For convenience of controller design, the following ROO is utilized to estimate the unmeasured state of uncertain systems (15) as

$$
\dot{\hat{z}}(t)=A_{11} \widehat{z}(t)+A_{12} \sigma(t),
$$

where the character $\widehat{z}(t)$ shows the estimation of unmeasured variables $z(t)$ and $\widehat{z}(t)=\widehat{\phi}_{\bar{z}}(t)=B^{\perp T} \widehat{\phi}(t)$ with $t \in[-\bar{d}, 0]$. With this observer, a prior knowledge of time-delays is not required. An error difference between the estimate state and the true state is defined by $e(t)$; that is, $e(t)=\widehat{z}(t)-z(t)$. Then, the time-delay observer error dynamics can be obtained from the first equation of system (15) and (30) as

$$
\begin{aligned}
\dot{e}(t)= & A_{11} e(t)+A_{11 d} e(t-d(t))-A_{11 d} \widehat{z}(t-d(t)) \\
& -A_{12 d} \sigma(t-d(t))-\Delta A_{11} z(t) \\
& -\Delta A_{11 d} z(t-d(t))-\Delta A_{12} \sigma(t) \\
& -\Delta A_{12 d} \sigma(t-d(t)),
\end{aligned}
$$

where $e(t)=\phi_{e}(t)=B^{\perp T}(\widehat{\phi}(t)-\phi(t)),-\bar{d} \leq t \leq 0$.

Remark 13. Here, we have attempted to extend the traditional Luenberger observer $[36,37]$ to generate a novel 
ROO scheme. The novel ROO design parameters should be proposed so that an asymptotically stable sliding mode will be generated on the sliding surface defined for uncertain systems with unknown time-varying delay. Also, the estimation error dynamics of observer asymptotically tend to zero in sliding mode. In other words, the invariance property will be ensured for ROO design. Further, the OFC in [4-7] were proposed based on FOO, which increases the computation and structure complexity. This full-dimension model is not necessary to implement. Consequently, the proposed ROO ensures that the conservatism is reduced, and the robustness is enhanced in comparison with FOO.

To determine an upper bound of governing error dynamic that supports the controller design, we establish the following novel Lemma 14.

Lemma 14. The matrix $A_{11}$ is a stable matrix and $\left\|\exp \left(A_{11} t\right)\right\| \leq k \exp \left(\lambda_{\max } t\right)$ for some $k>0$, where $\lambda_{\max }$ is a maximum eigenvalue of $A_{11}$.

Proof of Lemma 14. The matrix $A_{11}$ is stable if and only if there exists the positive-definite matrix $Q$ such that

$$
A_{11} Q+Q A_{11}^{T}<0
$$

Clearly, the following is valid when the LMI constraint (8) is feasible:

$$
\begin{aligned}
P & =\Gamma X \Gamma+B Y B^{T}>0, \\
B^{\perp T} P B^{\perp} & =B^{\perp T} \Gamma X \Gamma B^{\perp}>0 .
\end{aligned}
$$

With $A_{11}=B^{\perp T} A P B^{\perp}\left(B^{\perp T} P B^{\perp}\right)^{-1}$ and by choosing $Q=$ $\left(B^{\perp T} P B^{\perp}\right)>0$. along with the LMI (8) and (32) and using (33), we achieve

$$
B^{\perp T}\left[A P+P A^{T}\right] B^{\perp}<0 .
$$

which means that the matrix $A_{11}$ is a stable matrix. Its maximum eigenvalues $\lambda_{\max }$ are all negative and real. Thus, we can easily get $\left\|\exp \left(A_{11} t\right)\right\| \leq k \exp \left(\lambda_{\max } t\right)$ for some $k>0$.

Lemma 15. Let $r(t-d(t))$ be delayed function of $r(t)$. Assume $c \geq 0, w(t), h(t)$, and $g(t)$ are nonnegative valued continuous functions. If

$$
\begin{aligned}
\|r(t)\| w(t) \leq & c+\int_{0}^{t}\|r(\tau-d(\tau))\| h(\tau) w(\tau) \mathrm{d} \tau \\
& +\int_{0}^{t} g(\tau) \mathrm{d} \tau,
\end{aligned}
$$

then, for a constant $\beta>1$,

$$
\begin{aligned}
\|r(t)\| w(t) \leq & c \exp \{\beta f(t)\} \\
& +\int_{0}^{t} g(\tau) \exp \{\beta f(t)-\beta f(\tau)\} \mathrm{d} \tau,
\end{aligned}
$$

where $f(t)=\int_{0}^{t} h(\tau) \mathrm{d} \tau$.
Proof of Lemma 15. Let $s(t)=c+\int_{0}^{t}\|r(\tau-d(\tau))\| h(\tau) w(\tau) \mathrm{d} \tau+$ $\int_{0}^{t} g(\tau) \mathrm{d} \tau$, then we have $\|r(t)\| w(t) \leq s(t)$. According to Lemma 3 of [35], we get

$$
\begin{aligned}
& \|r(t-d(t))\| w(t) \leq \beta\|r(t)\| w(t) \leq \beta s(t) \\
& \text { for } \beta>1 ;
\end{aligned}
$$

Exploiting (37) and taking the time derivative of $s(t)$ yield $\dot{s}(t)=\|r(t-d(t))\| h(t) w(t)+g(t) \leq \beta h(t) s(t)+g(t)$, then $\{\dot{s}(t)-\beta h(t) s(t)\} \exp \{-\beta f(t)\} \leq g(t) \exp \{-\beta f(t)\}$. Because of $f(t)=\int_{0}^{t} h(\tau) \mathrm{d} \tau$, we have

$$
\frac{\mathrm{d}}{\mathrm{d} t}\{s(t) \exp \{-\beta f(t)\}\} \leq g(t) \exp \{-\beta f(t)\}
$$

Integrating the above inequality on both sides, we obtain

$$
\begin{aligned}
s(t) \leq & c \exp \{\beta f(t)\} \\
& +\int_{0}^{t} g(\tau) \exp \{\beta f(t)-\beta f(\tau)\} \mathrm{d} \tau .
\end{aligned}
$$

Since $\|r(t)\| w(t) \leq s(t)$, we can conclude that

$$
\begin{aligned}
\|r(t)\| w(t) \leq & c \exp \{\beta f(t)\} \\
& +\int_{0}^{t} g(\tau) \exp \{\beta f(t)-\beta f(\tau)\} \mathrm{d} \tau .
\end{aligned}
$$

Remark 16. This lemma is established to handle an unknown error of the observer error dynamics in the control design problem. It can be applied to a wider class of systems with time-delay, making it a valuable contribution to the field.

With the definition of $\Gamma$ in (7), generally, the design of FTOFC is designed for mismatched uncertain systems with unknown time-varying delay. In other words, if the term $B^{\perp T} D \neq 0$, then the uncertainties $\Delta A(t)$ and $\Delta A_{d}(t)$ will be pursued.

By utilizing (27), the dynamic error (31) can be reduced by the following form:

$$
\begin{aligned}
\dot{e}(t)= & A_{11} e(t)+A_{11 d} e(t-d(t))-A_{11 d} \widehat{z}(t-d(t)) \\
& -A_{12 d} \sigma(t-d(t))-\Delta A_{12} \sigma(t) \\
& -\Delta A_{12 d} \sigma(t-d(t))
\end{aligned}
$$

where $\widehat{e}(t)=\widehat{\phi}_{e}(t)=\widehat{\phi}_{\widehat{z}}(t)-\phi_{z}(t)=B^{\perp T}[\widehat{\phi}(t)-\phi(t)]$, with $t \in[-\bar{d}, 0]$. 
In order to prove a stability of error dynamic, we form a composite dynamical equation combining system (15) with (30) as

$$
\begin{aligned}
& {\left[\begin{array}{c}
\dot{z}(t) \\
\dot{\sigma}(t) \\
\dot{\bar{z}}(t)
\end{array}\right]=\left[\begin{array}{ccc}
A_{11}+\Delta A_{11} & A_{12}+\Delta A_{12} & 0 \\
A_{21}+\Delta A_{21} & A_{22}+\Delta A_{22} & 0 \\
0 & A_{12} & A_{11}
\end{array}\right]\left[\begin{array}{c}
z(t) \\
\sigma(t) \\
\widehat{z}(t)
\end{array}\right]} \\
& +\left[\begin{array}{ccc}
A_{11 d}+\Delta A_{11 d} & A_{12 d}+\Delta A_{12 d} & 0 \\
A_{21 d}+\Delta A_{21 d} & A_{22 d}+\Delta A_{22 d} & 0 \\
0 & 0 & 0
\end{array}\right]\left[\begin{array}{l}
z(t-d(t)) \\
\sigma(t-d(t)) \\
\widehat{z}(t-d(t))
\end{array}\right] \\
& +\left[\begin{array}{c}
0 \\
(S B) \\
0
\end{array}\right][u(t)+\xi(x(t), x(t-d(t)), t)], \\
& y(t)=\left[\begin{array}{lll}
C P B^{\perp}\left(B^{\perp T} P B^{\perp}\right)^{-1} & C B(S B)^{-1} & 0
\end{array}\right]\left[\begin{array}{l}
z(t) \\
\sigma(t) \\
\widehat{z}(t)
\end{array}\right],
\end{aligned}
$$

where $A_{11}+\Delta A_{11}, A_{11 d}+\Delta A_{11 d}, A_{12}+\Delta A_{12}, A_{12 d}+\Delta A_{12 d}$, $A_{21}+\Delta A_{21}, A_{21 d}+\Delta A_{21 d}, A_{22}+\Delta A_{22}$, and $A_{22 d}+\Delta A_{22 d}$ are defined as (13).

To discuss system behavior in the sliding mode, we introduce an equivalence transformation as

$$
\begin{aligned}
{\left[\begin{array}{l}
z(t) \\
\sigma(t) \\
e(t)
\end{array}\right] } & {\left[\begin{array}{c}
z(t) \\
\sigma(t) \\
\widehat{z}(t)-z(t)
\end{array}\right] } \\
& =\left[\begin{array}{ccc}
I & 0 & 0 \\
0 & I & 0 \\
-I & 0 & I
\end{array}\right]\left[\begin{array}{l}
z(t) \\
\sigma(t) \\
\widehat{z}(t)
\end{array}\right], \\
{\left[\begin{array}{c}
z(t-d(t)) \\
\sigma(t-d(t)) \\
e(t-d(t))
\end{array}\right] } & {\left[\begin{array}{c}
z(t-d(t)) \\
\widehat{z}(t-d(t))-z(t-d(t))
\end{array}\right] } \\
& =\left[\begin{array}{ccc}
I & 0 & 0 \\
0 & I & 0 \\
-I & 0 & I
\end{array}\right]\left[\begin{array}{l}
z(t-d(t)) \\
\sigma(t-d(t)) \\
\widehat{z}(t-d(t))
\end{array}\right] .
\end{aligned}
$$

Hence, it can be seen that the system dynamical equation (42) in the sliding mode is

$$
\begin{aligned}
{\left[\begin{array}{c}
\dot{z}(t) \\
\dot{e}(t)
\end{array}\right]=} & {\left[\begin{array}{cc}
A_{11} & 0 \\
0 & A_{11}
\end{array}\right]\left[\begin{array}{l}
z(t) \\
e(t)
\end{array}\right] } \\
& +\left[\begin{array}{cc}
A_{11 d} & 0 \\
0 & A_{11 d}
\end{array}\right]\left[\begin{array}{l}
z(t-d(t)) \\
e(t-d(t))
\end{array}\right] \\
& +\left[\begin{array}{cc}
\Delta A_{11} & 0 \\
0 & 0
\end{array}\right]\left[\begin{array}{l}
z(t) \\
e(t)
\end{array}\right]
\end{aligned}
$$

$$
\begin{aligned}
& +\left[\begin{array}{cc}
\Delta A_{11 d} & 0 \\
0 & 0
\end{array}\right]\left[\begin{array}{l}
z(t-d(t)) \\
e(t-d(t))
\end{array}\right] \\
& -\left[\begin{array}{cc}
0 & 0 \\
0 & A_{11 d}
\end{array}\right]\left[\begin{array}{l}
z(t-d(t)) \\
\hat{z}(t-d(t))
\end{array}\right], \\
& y(t)=\left[C P B^{\perp}\left(B^{\perp T} P B^{\perp}\right)^{-1} 0\right]\left[\begin{array}{l}
z(t) \\
e(t)
\end{array}\right] .
\end{aligned}
$$

Remark 17. According to Lemma 14, the reduced-order observer (30) is asymptotically stable in sliding mode, $\sigma(t)=$ $\sigma(t-d(t))=0$, which means that $\widehat{z}(t-d(t))$ in (41) also converges to zero. Thus, one can see that the error dynamics (41) and (44) are asymptotically stable in the sliding mode; that is, the mismatched uncertain system (15) is asymptotically stable, and the invariance property is ensured by ROO. Further, the stability examination of an overall closed-loop system in sliding mode is concluded in Remark 12.

In order to use the estimated variables and observer error in controller design, we establish Theorem 18.

Theorem 18. Let error $e(0)$ be an initial condition of the error $e(t)$. The norm of estimation error $\|e(t)\|$ is bounded by $\eta(t)$ for all time. The term $\eta(t)$ is the solution of

$$
\begin{aligned}
& \dot{\eta}(t)=\lambda \eta(t) \\
& \quad+k \beta\left[\left(\beta_{1}\left\|A_{12 d}\right\|+\left\|B^{\perp T} D\right\|\left\|E B(S B)^{-1}\right\|\left(1+\beta_{1}\right)\right)\right. \\
& \left.\cdot\|\sigma(t)\|+\beta_{2}\left\|A_{11 d}\right\|\|\widehat{z}(t)\|\right],
\end{aligned}
$$

where $\eta(0) \geq k\|e(0)\|, \lambda=\lambda_{\max }+k \beta\left\|A_{11 d}\right\|<0$, and $\lambda_{\max }$ is the maximum eigenvalue of $A_{11}$.

Proof of Theorem 18. Based on Lemma 14, we obtain the norm dynamic error solved from (41) as

$$
\begin{aligned}
& \|e(t)\| \leq\left\|\exp \left(A_{11} t\right)\right\|\|e(0)\| \\
& +\int_{0}^{t}\left\|\exp \left[A_{11}(t-\tau)\right]\right\|\left[\left\|A_{12 d} \sigma(\tau-d(\tau))\right\|\right. \\
& +\left\|A_{11 d} e(\tau-d(\tau))\right\|+\left\|A_{11 d} \widehat{z}(\tau-d(\tau))\right\| \\
& \left.+\left\|\Delta A_{12} \sigma(\tau)\right\|+\left\|\Delta A_{12 d} \sigma(\tau-d(\tau))\right\|\right] \mathrm{d} \tau, \\
& \|e(t)\| \leq k\|e(0)\| \exp \left(\lambda_{\max } t\right)+\int_{0}^{t} k \\
& \cdot \exp \left[\lambda_{\max }(t-\tau)\right]\left[\left\|A_{12 d}\right\|\|\sigma(\tau-d(\tau))\|\right. \\
& +\left\|A_{11 d}\right\|\|e(\tau-d(\tau))\|+\left\|A_{11 d}\right\|\|\widehat{z}(\tau-d(\tau))\| \\
& +\left\|B^{\perp T} D\right\|\left\|E B(S B)^{-1}\right\| \\
& \quad \cdot(\|\sigma(\tau)\|+\|\sigma(\tau-d(\tau))\|)] \mathrm{d} \tau .
\end{aligned}
$$


We multiply both sides by the term $\exp \left(-\lambda_{\max } t\right)$ for the above inequality; then

$$
\begin{aligned}
& \|e\| \exp \left(-\lambda_{\max } t\right) \leq k\|e(0)\|+\int_{0}^{t} k \exp \left(-\lambda_{\max } \tau\right) \\
& \cdot\left\|A_{11 d}\right\|\|e(\tau-d(\tau))\| \mathrm{d} \tau+\int_{0}^{t} k \exp \left(-\lambda_{\max } \tau\right) \\
& \quad \times\left[\left\|B^{\perp T} D\right\|\left\|E B(S B)^{-1}\right\|\|\sigma(\tau)\|\right. \\
& +\left\|A_{12 d}\right\|\|\sigma(\tau-d(\tau))\|+\left\|A_{11 d}\right\|\|\bar{z}(\tau-d(\tau))\| \\
& \left.+\left\|B^{\perp T} D\right\|\left\|E B(S B)^{-1}\right\|\|\sigma(\tau-d(\tau))\|\right] \mathrm{d} \tau .
\end{aligned}
$$

Let

$$
\begin{aligned}
& \|r(t)\|=\|e(t)\|, \\
& w(t)=\exp \left(-\lambda_{\max } t\right), \\
& c=k\|e(0)\|, \\
& \|r(t-d(t))\|=\|e(t-d(t))\|, \\
& h(t)=k\left\|A_{11 d}\right\|, \\
& \beta>1, \\
& f(t)=\int_{0}^{t} h(\tau) \mathrm{d} \tau=k\left\|A_{11 d}\right\| t, \\
& g(t)=k \exp \left(-\lambda_{\max } t\right)\left[\left\|B^{\perp T} D\right\|\left\|E B(S B)^{-1}\right\|\|\sigma(t)\|\right. \\
& \quad+\left\|A_{12 d}\right\|\|\sigma(t-d(t))\|+\left\|A_{11 d}\right\|\|\bar{z}(t-d(t))\| \\
& \left.\quad+\left\|B^{\perp T} D\right\|\left\|E B(S B)^{-1}\right\|\|\sigma(t-d(t))\|\right] .
\end{aligned}
$$

Applying Lemma 15, we obtain

$$
\begin{aligned}
& \|e(t)\| \exp \left(-\lambda_{\max } t\right) \leq k\|e(0)\| \exp \left(k \beta\left\|A_{11 d}\right\| t\right) \\
& \quad+\int_{0}^{t} k \beta \exp \left(-\lambda_{\max } \tau\right) \\
& \quad \times\left[\left\|B^{\perp T} D\right\|\left\|E B(S B)^{-1}\right\|\|\sigma(\tau)\|\right. \\
& +\left\|A_{12 d}\right\|\|\sigma(\tau-d(\tau))\|+\left\|A_{11 \mathrm{~d}}\right\|\|\bar{z}(\tau-d(\tau))\| \\
& \left.+\left\|B^{\perp T} D\right\|\left\|E B(S B)^{-1}\right\|\|\sigma(\tau-d(\tau))\|\right] \\
& \quad \cdot \exp \left(k \beta\left\|A_{11 d}\right\| t-k \beta\left\|A_{11 d}\right\| \tau\right) \mathrm{d} \tau .
\end{aligned}
$$

Shift $\exp \left(-\lambda_{\max } t\right)$ to the right-hand side term of inequality (49) and use the Lemma 3 of [35]; that is, $\|\sigma(t-d(t))\| \leq$ $\beta_{1}\|\sigma(t)\|,\|\widehat{z}(t-d(t))\| \leq \beta_{2}\|\widehat{z}(t)\|$ with $\beta_{1}>1, \beta_{2}>1$. It can be evaluated as

$$
\begin{gathered}
\|e(t)\| \leq \eta(0) \exp \left[\left(\lambda_{\max }+k \beta\left\|A_{11 d}\right\|\right) t\right]+\int_{0}^{t} k \beta \\
\cdot \exp \left(\lambda_{\max }+k \beta\left\|A_{11 d}\right\|\right)(t-\tau) \times\left[\left\|A_{12 d}\right\|\right.
\end{gathered}
$$

$$
\begin{gathered}
\cdot\|\sigma(\tau-d(\tau))\|+\left\|B^{\perp T} D\right\|\left\|E B(S B)^{-1}\right\|\|\sigma(\tau)\| \\
+\left\|A_{11 d}\right\|\|\bar{z}(\tau-d(\tau))\|+\left\|B^{\perp T} D\right\|\left\|E B(S B)^{-1}\right\| \\
\cdot\|\sigma(\tau-d(\tau))\|] \mathrm{d} \tau, \\
\|e(t)\| \leq \eta(0) \exp \left[\left(\lambda_{\max }+k \beta\left\|A_{11 d}\right\|\right) t\right]+\int_{0}^{t} k \beta \\
\cdot \exp \left(\lambda_{\max }+k \beta\left\|A_{11 d}\right\|\right)(t-\tau) \\
\times\left[\left(\beta_{1}\left\|A_{12 d}\right\|+\left\|B^{\perp T} D\right\|\left\|E B(S B)^{-1}\right\|\left(1+\beta_{1}\right)\right)\right. \\
\left.\cdot\|\sigma(\tau)\|+\beta_{2}\left\|A_{11 d}\right\|\|\widehat{z}(\tau)\|\right] \mathrm{d} \tau=\eta(t),
\end{gathered}
$$

where $\eta(t)$ satisfies (45). Hence, we can see that $\|e(t)\| \leq \eta(t)$ for all time. Thus, the proof of Theorem 18 is finished.

Now, we design control input $u(t)$ in system (15); the control input will be appropriately designed with the help of the ROO tool (30).

Theorem 19. Consider that the unmeasured states of the mismatched uncertain time-varying systems (15) are estimated by the ROO (30) and the error dynamic (41) satisfies Theorem 18. Under Assumptions 1-4, system (15) can be moved onto the sliding surface in finite time and maintains a sliding motion on it, thereafter, by the following control law:

$$
u(t)=-\bar{k}_{1} \sigma(t)-\left\{\bar{k}_{2}[\|\bar{z}(t)\|+\eta(t)]+\bar{k}_{3}\right\} \frac{\sigma(t)}{\|\sigma(t)\|} ;
$$

if the control gains are chosen as

$$
\begin{aligned}
\bar{k}_{1} & >(S B)^{-1}\left(\left\|A_{22}\right\|+\beta_{1}\left\|A_{22 d}\right\|\right)+\left(1+\beta_{1}\right)(S B)^{-1} \\
& \times\left[\left\|N B^{T} P^{-1} D\right\|\left\|E B(S B)^{-1}\right\|\right. \\
& \left.+k_{m}\|S B\|\left\|B(S B)^{-1}\right\|\right], \\
\bar{k}_{2} & >(S B)^{-1}\left[\left\|A_{21}\right\|+\varepsilon\left\|A_{21 d}\right\|\right. \\
& +(\varepsilon+1)\left\|N B^{T} P^{-1} D\right\|\left\|E P B^{\perp}\left(B^{\perp T} P B^{\perp}\right)^{-1}\right\| \\
& \left.+k_{m}(\varepsilon+1)\|S B\|\left\|P B^{\perp}\left(B^{\perp T} P B^{\perp}\right)^{-1}\right\|\right], \\
\bar{k}_{3} & >(S B)^{-1}\left(k_{\xi}\|S B\|+\alpha\right),
\end{aligned}
$$

for any scalar $\alpha>0$, where $\widehat{z}(t)$ is solution of $(30), \eta(t)$ is found in Theorem 18, and $\bar{k}_{1}, \bar{k}_{2}, \bar{k}_{3}$ are constant gains.

Proof of Theorem 19. State transformation $T$ in (12) implies that

$$
\begin{aligned}
x(t)= & P B^{\perp}\left(B^{\perp T} P B^{\perp}\right)^{-1} z(t) \\
& +B(S B)^{-1} \sigma(t),
\end{aligned}
$$




$$
\begin{aligned}
x(t-d(t))= & P B^{\perp}\left(B^{\perp T} P B^{\perp}\right)^{-1} z(t-d(t)) \\
& +B(S B)^{-1} \sigma(t-d(t))
\end{aligned}
$$

because $z(t)=\widehat{z}(t)-e(t), z(t-d(t))=\widehat{z}(t-d(t))-e(t-d(t))$, and $\|e(t)\| \leq \eta(t)$. In addition, it follows from Lemma 3 of [35] that it is clear that $\|z(t-d(t))\| \leq \varepsilon\|z(t)\|,\|\sigma(t-d(t))\| \leq$ $\beta_{1}\|\sigma(t)\|$ for some scalars $\varepsilon>1, \beta_{1}>1$. Therefore, (53) can be rewritten as

$$
\begin{aligned}
& \|x(t)\| \\
& \leq\left\|P B^{\perp}\left(B^{\perp T} P B^{\perp}\right)^{-1}\right\|(\|\widehat{z}(t)\|+\eta(t)) \\
& \quad+\left\|B(S B)^{-1}\right\|\|\sigma(t)\|, \\
& \|x(t-d(t))\| \\
& \leq \varepsilon\left\|P B^{\perp}\left(B^{\perp T} P B^{\perp}\right)^{-1}\right\|(\|\widehat{z}(t)\|+\eta(t)) \\
& \quad+\beta_{1}\left\|B(S B)^{-1}\right\|\|\sigma(t)\| .
\end{aligned}
$$

Let us consider the function $V(\sigma(t))=0.5 \sigma^{T}(t) \sigma(t)$. If we differentiate $V(\sigma(t))$ with respect to time and combine with the second equation of system (15), then

$$
\begin{aligned}
& \sigma^{T}(t) \dot{\sigma}(t)=\sigma^{T}(t)\left\{\left[A_{21}+\Delta A_{21}\right] z(t)\right. \\
&+ {\left[A_{21 d}+\Delta A_{21 d}\right] z(t-d(t))+\left[A_{22}+\Delta A_{22}\right] } \\
& \cdot \sigma(t)+\left[A_{22 d}+\Delta A_{22 d}\right] \sigma(t-d(t))+(S B) \\
&\cdot[u(t)+\xi(x(t), x(t-d(t)), t)]\} . \\
& \sigma^{T}(t) \dot{\sigma}(t) \leq\|\sigma(t)\| \\
& \quad \cdot\left\{\left[\left\|A_{21}\right\|+\left\|N B^{T} P^{-1} D\right\|\left\|E P B^{\perp}\left(B^{\perp T} P B^{\perp}\right)^{-1}\right\|\right]\right. \\
& \quad \cdot[\|\widehat{z}(t)\|+\eta(t)] \\
&+\varepsilon\left[\left\|A_{21 d}\right\|+\left\|N B^{T} P^{-1} D\right\|\left\|E P B^{\perp}\left(B^{\perp T} P B^{\perp}\right)^{-1}\right\|\right] \\
& \quad \cdot[\|\widehat{z}(t)\|+\eta(t)] \\
&+\left[\left\|A_{22}\right\|+\left\|N B^{T} P^{-1} D\right\|\left\|E B(S B)^{-1}\right\|\right] \sigma(t) \\
&\left.+\beta_{1}\left[\left\|A_{22 d}\right\|+\left\|N B^{T} P^{-1} D\right\|\left\|E B(S B)^{-1}\right\|\right]\|\sigma(t)\|\right\} \\
&+\sigma^{T}(S B) u(t)+\|\sigma\|\|S B\|\left\{k_{\xi}\right. \\
&\left.+k_{m}[\|x(t)\|+\|x(t-d(t))\|]\right\},
\end{aligned}
$$

Substituting controller (51) and gains (52) and (54) into inequality (55), we get $\sigma^{T}(t) \dot{\sigma}(t) \leq-\alpha\|\sigma(t)\|$. Thus, we proved that the finite-time of system (15) converges toward the sliding surface $\sigma(t)=0$ and subsequently remains on it. This completes the proof of Theorem 19.
Remark 20. It should be noted that the proposed control laws (51) uses the measured output information completely in the control design and the estimated variables estimated by the ROO tool (30). In addition, we can see that when time-delay $d(t)$ is an unknown, controller (51) is applicable. Therefore, the proposed controller does not need the availability of the system states, and this study provides a methodology to reduce conservatism and enhance robustness.

4.2. Summary of Design Algorithm. For the mismatched uncertain systems with unknown time-varying delay, the sliding surface in (6) and the proposed FTOFC can be simultaneously designed by the following steps.

Step 1. Solving the LMI (8) obtains the matrix solutions $(X, Y)$ and computing the sliding matrix $F$ according to (9).

Step 2. Substituting matrix $F$ into equation (6), the sliding function $\sigma(t)$ is found.

Step 3. The ROO $\widehat{z}(t)$ is designed as (30).

Step 4. The FTOFC is synthesized as follows. First, determine the upper bound of observer dynamic error $\eta(t)$ as (45). Then, design the FTOFC $u(t)$ according to (51).

\section{Numerical Example}

In this simulation study, we apply the proposed control scheme, which is designed based on ROO tool, to the mismatched uncertain systems. The mathematical representation of a system subject to unknown time-varying delay and external disturbances is taken from [33]

$$
\begin{aligned}
\dot{x}(t)= & {\left[\left[\begin{array}{ccc}
0 & 2 & 0 \\
1 & 2 & 0 \\
2 & 1 & -2
\end{array}\right]+\Delta A(t)\right] x(t) } \\
& +\left[\left[\begin{array}{ccc}
0 & 2 & -1 \\
0 & 1 & -1 \\
0 & -1 & 1
\end{array}\right]+\Delta A_{d}(t)\right] x(t-d(t)) \\
& +\left[\begin{array}{c}
0 \\
1 \\
-0.5
\end{array}\right]\left[\begin{array}{c}
u(t)+\xi(x(t), x(t-d(t)), t)], \\
y(t)=
\end{array}\right. \\
& C x(t)=\left[\begin{array}{lll}
1 & 1 & 0
\end{array}\right] x(t),
\end{aligned}
$$

where the terms $\Delta A(t)$ and $\Delta A_{d}(t)$ are uncertainties but bounded by time variables ranging in $[-1,1]$. The initial condition of system states was given as $\left[\begin{array}{lll}-0.1 & 0.15 & 0.2\end{array}\right]^{T}$. The matrices of parameter uncertainties are $D=\left[\begin{array}{lll}0 & 1 & 0\end{array}\right]^{T}, E=$ $\left[\begin{array}{lll}1 & 1 & 0\end{array}\right]$, and the external perturbations input is $\| \xi(x(t), x(t-$ $d(t)), t) \| \leq 0.2(\|x(t)\|+\|x(t-d(t))\|)$. For purpose of simulation, let the unknown time-varying delay be $d(t)=$ $0.15(1+\sin 0.5 t)[20]$. Based on the above data, we can see that the system does not need to satisfy the so-called matching 


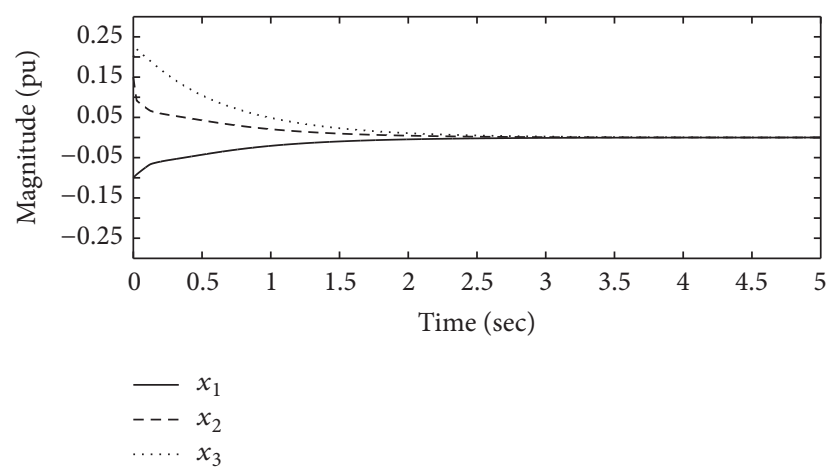

FIGURE 1: Closed-loop response of time-delay uncertain system.

condition. First, using MATLAB's LMI Control Toolbox, we can find the feasible solutions of the LMIs (8) as

$$
\begin{aligned}
& X=\left[\begin{array}{ccc}
-198.3491 & -185.7861 & -2.9044 \\
-185.7861 & -171.8494 & -3.1133 \\
-2.9044 & -3.1133 & 0.5068
\end{array}\right], \\
& Y=0.6806 .
\end{aligned}
$$

Next, solving formula (9) via the results of (57), the matrix is selected to be $N=I$. The corresponding sliding function for system (56) is described by

$$
\sigma(x(t))=F y(t)=[1.4693] y(t) .
$$

According to (30), the suitable ROO is given by

$$
\dot{\vec{z}}(t)=\left[\begin{array}{cc}
-1.8211 & -0.0894 \\
0.3578 & -2.1789
\end{array}\right] \widehat{z}(t)+\left[\begin{array}{c}
-0.4008 \\
2.2422
\end{array}\right] \sigma(t) .
$$

Thus, based on Theorem 19, the control signal is synthesized as follows:

$$
\begin{aligned}
u(t)= & -7.1104 \sigma(t) \\
& -\{8.2151[\|\widehat{z}(t)\|+\eta(t)]+0.0010\} \frac{\sigma(t)}{\|\sigma(t)\|},
\end{aligned}
$$

where the estimated variables $\widehat{z}(t)$ are solutions of observer (59) and $\eta(t)$ is the solution of $\dot{\eta}(t)=-1.9997 \eta(t)+$ $0.6089\|\sigma(t)\|+2.8583 e^{-04}\|\widehat{z}(t)\|$. The simulation results are depicted in Figures 1-4, which verify the effectiveness of the proposed method.

Remark 21. From Figures 1 and 2, we can see that closedloop system states of the time-varying delay systems decline to zero immediately under the proposed FTOFC based on ROO and state estimate error of the designed ROO is asymptotically stable. According to Figure 3, it can be observed that control signal is convergent. Besides, the time evolution of the sliding function was exposed in Figure 4. Based on these simulation results, we can see that the proposed controller is effective in dealing with mismatched uncertainties, and the system has a good performance. It should be pointed out that the controllers in the above published works are not designed for this problem.

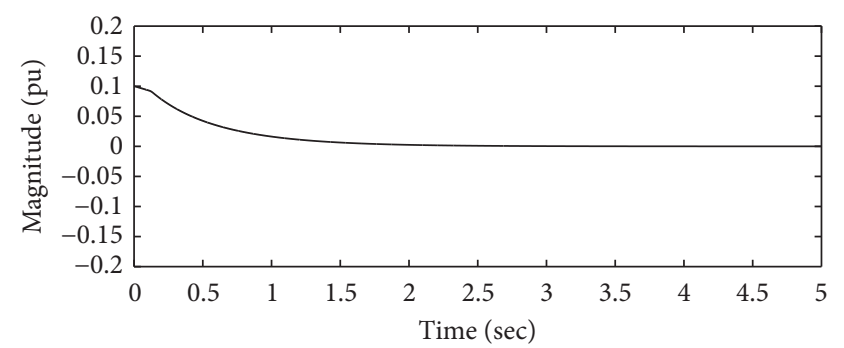

Figure 2: Time response of error dynamic.

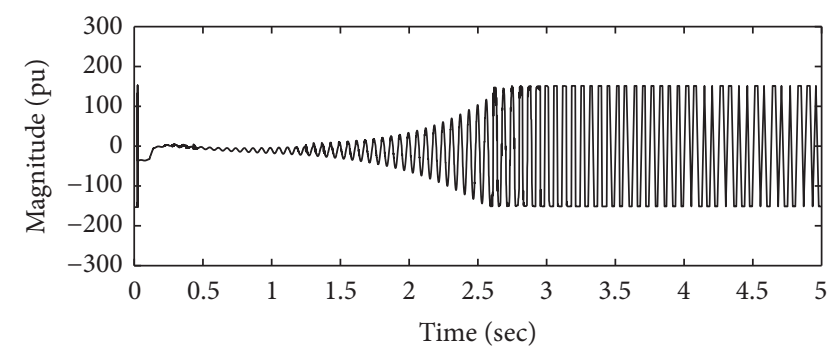

Figure 3: Control input signal of the system.

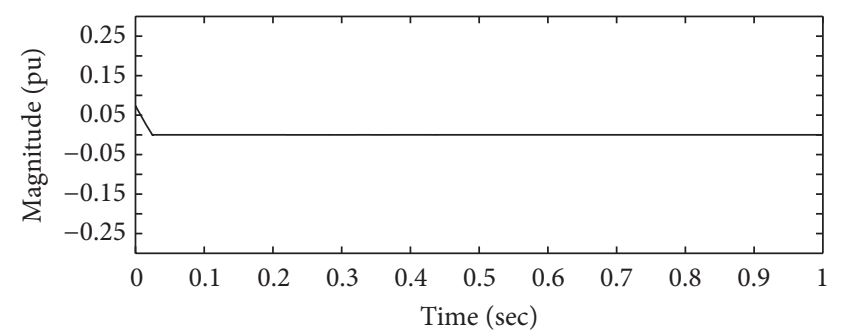

FIGURE 4: The trajectory of the sliding function.

\section{Conclusions}

This paper represents the novel VSC design, which includes the finite-time convergence sliding mode, invariance property, asymptotic stability, and output variables only, for the unknown time-varying delay systems. We have established the novel lemma for a wider class of systems with timedelay. Based on the ROO tool and the Moore-Penrose inverse technique, the novel FTOFC has been designed for the mismatched uncertain systems with unknown time-varying delay. A necessary and sufficient condition of the sliding surface existence is vital for ensuring a desired system response has been obtained. The proposed method has eliminated the restrictions required in existing studies. Moreover, this work could provide a methodology for reducing conservatism and enhancing robustness for uncertain systems with unknown time-varying delay using the ROO tool in control design. Furthermore, employing the Lyapunov stability theory and the LMI approach, the dynamic of the reduction order system is asymptotically stable under sufficient condition developed and guarantees the invariance property in sliding mode. Finally, the results obtained are supported by a numerical example. 


\section{Conflicts of Interest}

The authors declare that there are no conflicts of interest regarding the publication of this paper.

\section{Acknowledgments}

The authors would like to thank the financial support provided by the Da-Yeh University in Taiwan.

\section{References}

[1] J. Y. Hung, W. Gao, and J. C. Hung, "Variable structure control: a survey," IEEE Transactions on Industrial Electronics, vol. 40, no. 1, pp. 2-22, 1993.

[2] S. H. Zak, Systems and Control, Oxford Univ. Press, Novel York, 2003.

[3] A. W. Olbrot, "A sufficiently large time delay in feedback loop must destroy exponential stability of any decay rate," Institute of Electrical and Electronics Engineers. Transactions on Automatic Control, vol. 29, no. 4, pp. 367-368, 1984.

[4] H. Li, P. Shi, D. Yao, and L. Wu, "Observer-based adaptive sliding mode control for nonlinear Markovian jump systems," Automatica, vol. 64, pp. 133-142, 2016.

[5] Z. Liu, L. Zhao, Y. Kao, and C. Gao, "Robust passive control for a class of uncertain neutral systems based on sliding mode observer," ISA Transactions, 2017.

[6] H.-C. Ting, J.-L. Chang, and Y.-P. Chen, "Applying output feedback integral sliding mode controller to uncertain timedelay systems with mismatched disturbances," International Journal of Control, Automation and Systems, vol. 9, no. 6, pp. 1056-1066, 2011.

[7] Y. Zhang, S. Xu, and Y. Chu, "Sliding mode observer-controller design for uncertain Markovian jump systems with time delays," International Journal of Robust and Nonlinear Control, vol. 22, no. 4, pp. 355-368, 2012.

[8] P. Zhang, Y. Kao, J. Zhu, and W. Li, " $\mathrm{H}_{\infty}$ observer-based sliding mode control for uncertain stochastic systems with timevarying delays," Mathematical Problems in Engineering, vol. 2013, Article ID 482430, 8 pages, 2013.

[9] I. Boulaabi, A. Sellami, and F. B. Hmida, "A robust sensor fault reconstruction based on a new sliding mode observer design method for uncertain delayed systems: a satellite system application," Control Engineering and Applied Informatics, vol. 17, no. 3, pp. 88-97, 2015.

[10] C.-T. Chen and S.-T. Peng, "A sliding mode control scheme for non-minimum phase non-linear uncertain input-delay chemical processes," Journal of Process Control, vol. 16, no. 1, pp. 37-51, 2006.

[11] A. Rezoug, B. Tondu, M. Hamerlain, and M. Tadjine, "RBFNNHOMS Non-singular Terminal Sliding Control of $n$-DOF Robotic Manipulator," Journal of Control Engineering and Applied Informatics, vol. 18, no. 3, pp. 52-62, 2016.

[12] S. C. Garcia, M. C. Teixeira, J. P. F. Garcia, U. N. Alves, and J. M. Ribeiro, "Discrete-time sliding mode control for uncertain networked system subject to time delay," Mathematical Problems in Engineering, vol. 2015, Article ID 195120, 10 pages, 2015.

[13] M. Darouach, "Reduced-order observers for linear neutral delay systems," Institute of Electrical and Electronics Engineers. Transactions on Automatic Control, vol. 50, no. 9, pp. 1407-1413, 2005.
[14] E. M. Jafarov, "Design modification of sliding mode observers for uncertain MIMO systems without and with time-delay," Asian Journal of Control, vol. 7, no. 4, pp. 380-392, 2005.

[15] W.-J. Liu, "State observer design for non-linear uncertain timevarying delay systems with unmatched uncertainties," Transactions of the Institute of Measurement and Control, vol. 33, no. 2, pp. 207-222, 2011.

[16] H. M. Trinh, P. S. Teh, and T. L. Fernando, “Time-delay systems: design of delay-free and low-order observers," Institute of Electrical and Electronics Engineers. Transactions on Automatic Control, vol. 55, no. 10, pp. 2434-2438, 2010.

[17] X.-G. Yan, S. K. Spurgeon, and C. Edwards, "State and parameter estimation for nonlinear delay systems using sliding mode techniques," Institute of Electrical and Electronics Engineers. Transactions on Automatic Control, vol. 58, no. 4, pp. 1023-1029, 2013.

[18] S. Boyd, L. El Ghaoui, E. Feron, and V. Balakrishnan, Linear matrix inequalities in system and control theory, Society for Industrial and Applied Mathematics (SIAM), Philadelphia, pa, USA, 1994.

[19] P. Gahinet, A. Nemirovskii, A. J. Laub, and M. Chilali, LMI Control Toolbox, Natick, MA: The MathWorks, Inc, 1994.

[20] A. Seuret, T. Floquet, J.-P. Richard, and S. K. Spurgeon, "A sliding mode observer for linear systems with unknown time varying delay," in Proceedings of the IEEE ACC'0, pp. 4558-4563, July 2007.

[21] C. E. de Souza, R. M. Palhares, and P. L. Peres, "Robust $\mathrm{H}_{\infty}$ filter design for uncertain linear systems with multiple time-varying state delays," IEEE Transactions on Signal Processing, vol. 49, no. 3, pp. 569-576, 2001.

[22] E. Fridman, U. Shaked, and L. Xie, "Robust $\mathrm{H}_{\infty}$ Filtering of linear systems with time-varying delay," Institute of Electrical and Electronics Engineers. Transactions on Automatic Control, vol. 48, pp. 159-165, 2003.

[23] Q. Gao, G. Feng, L. Liu, J. Qiu, and Y. Wang, "An ISMC approach to robust stabilization of uncertain stochastic time-delay systems," IEEE Transactions on Industrial Electronics, vol. 61, no. 12, pp. 6986-6994, 2014.

[24] F. Gouaisbaut, M. Dambrine, and J. P. Richard, "Robust control of delay systems: a sliding mode control design via LMI," Systems and Control Letters, vol. 46, pp. 219-230, 2002.

[25] X. Han, E. Fridman, and S. K. Spurgeon, "Sliding-mode control of uncertain systems in the presence of unmatched disturbances with applications," International Journal of Control, vol. 83, no. 12, pp. 2413-2426, 2010.

[26] S. C. Liu and S. F. Lin, "Robust sliding control for mismatched uncertain fuzzy time-delay systems using linear matrix inequality approach," Journal of the Chinese Institute of Engineers, vol. 36, no. 5, pp. 589-597, 2013.

[27] H. Xing, C. C. Gao, and D. Li, "Sliding mode variable structure control for parameter uncertain stochastic systems with timevarying delay," Journal of Mathematical Analysis and Applications, vol. 355, no. 2, pp. 689-699, 2009.

[28] Y. Niu and D. W. Ho, "Robust observer design for Itô stochastic time-delay systems via sliding mode control," Systems and Control Letters, vol. 55, pp. 781-793, 2006.

[29] Y. W. Tsai and V. V. Huynh, "Output feedback and single-phase sliding mode control for complex interconnected systems," Mathematical Problems in Engineering, vol. 2015, Article ID 946385, 16 pages, 2015. 
[30] S. Sayyaddelshad and T. Gustafsson, " $\mathrm{H}_{\infty}$ observer design for uncertain nonlinear discrete-time systems with time-delay: LMI optimization approach," International Journal of Robust and Nonlinear Control, vol. 25, no. 10, pp. 1514-1527, 2015.

[31] H. H. Choi, "An LMI-based switching surface design method for a class of mismatched uncertain systems," Institute of Electrical and Electronics Engineers. Transactions on Automatic Control, vol. 48, no. 9, pp. 1634-1638, 2003.

[32] H. H. Choi, "Variable structure output feedback control design for a class of uncertain dynamic systems," Automatica, vol. 38, no. 2, pp. 335-341, 2002.

[33] J. Lian, J. Zhao, and G. M. Dimirovski, "Robust $\mathrm{H}_{\infty}$ sliding mode control for a class of uncertain switched delay systems," International Journal of Systems Science, vol. 40, no. 8, pp. 855866, 2009.

[34] J. Lian and J. Zhao, "Output feedback variable structure control for a class of uncertain switched systems," Asian Journal of Control, vol. 11, no. 1, pp. 31-39, 2009.

[35] X. G. Yan, S. K. Spurgeon, and C. Edwards, "Static output feedback sliding mode control for time-varying delay systems with time-delayed nonlinear disturbances," International Journal of Robust and Nonlinear Control, vol. 20, no. 7, pp. 777-788, 2010.

[36] I. Haskara, U. Ozguner, and V. Utkin, "On variable structure observers," in Proceedings of the 1996 IEEE International Workshop on Variable Structure Systems, VSS'96, pp. 193-198, December 1996.

[37] D. G. Luenberger, “An Introduction to Observers," IEEE Transactions on Automatic Control, vol. 16, no. 6, pp. 596-602, 1971. 


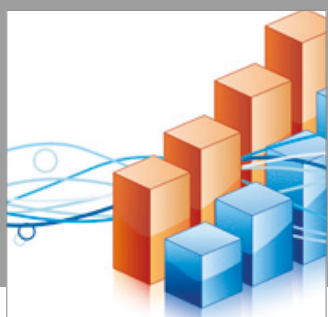

Advances in

Operations Research

vatersals

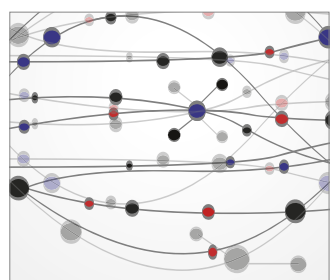

\section{The Scientific} World Journal
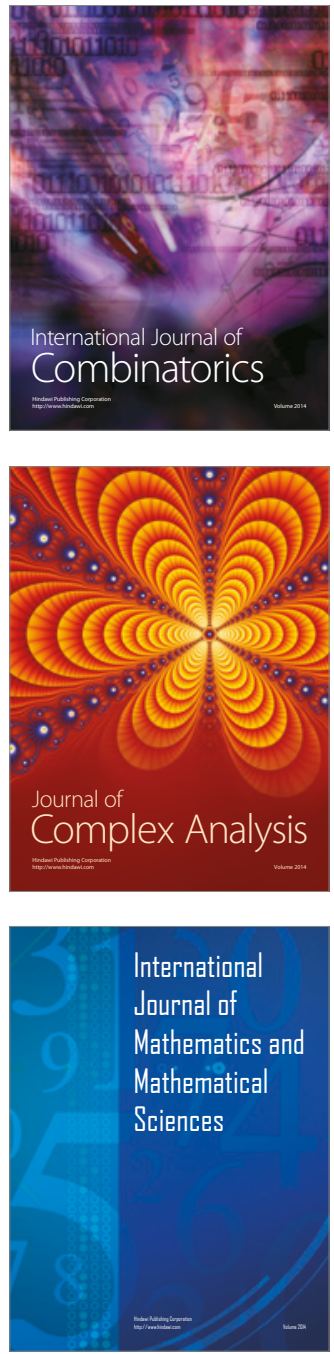
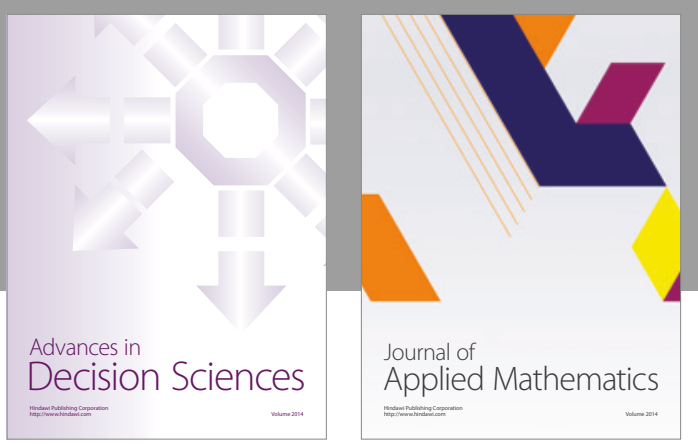

Algebra

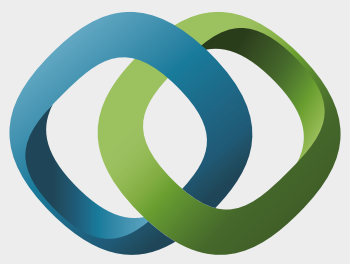

\section{Hindawi}

Submit your manuscripts at

https://www.hindawi.com
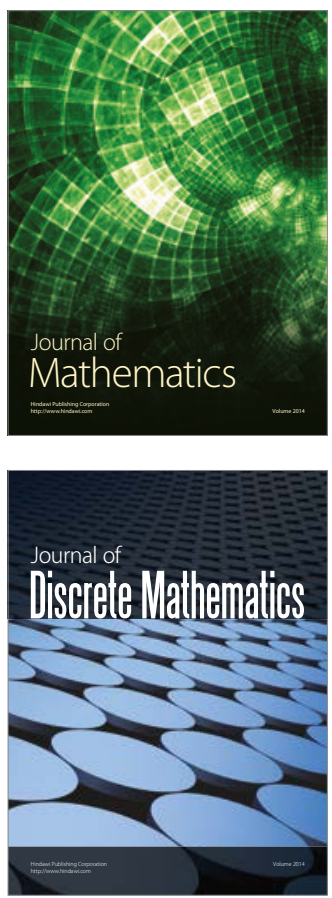

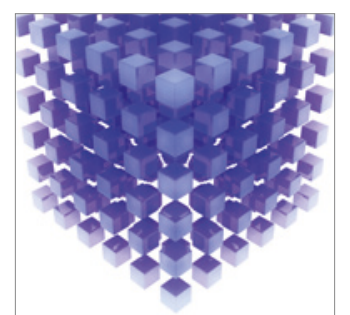

Mathematical Problems in Engineering
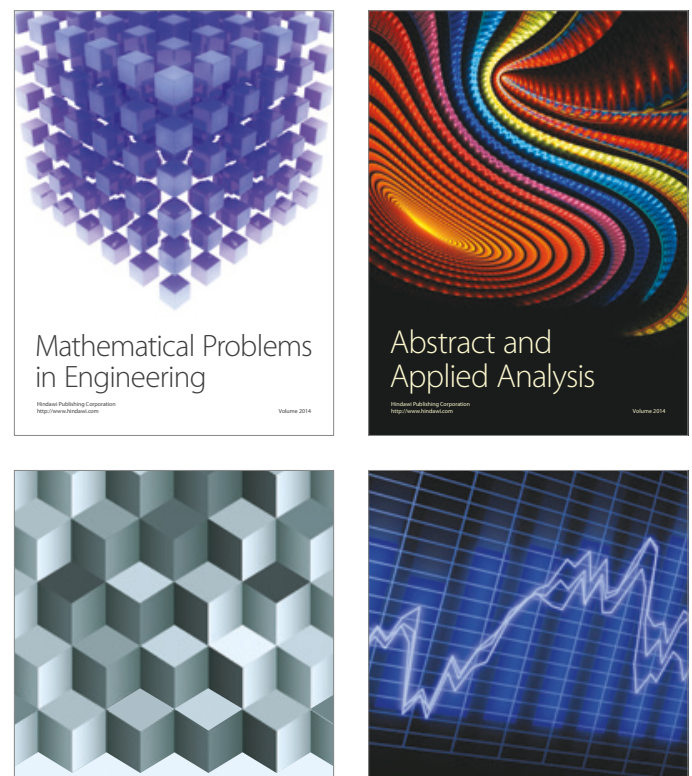

Journal of

Function Spaces

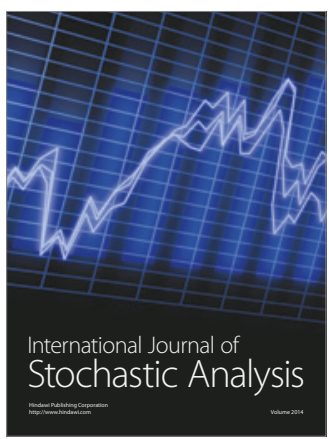

Probability and Statistics
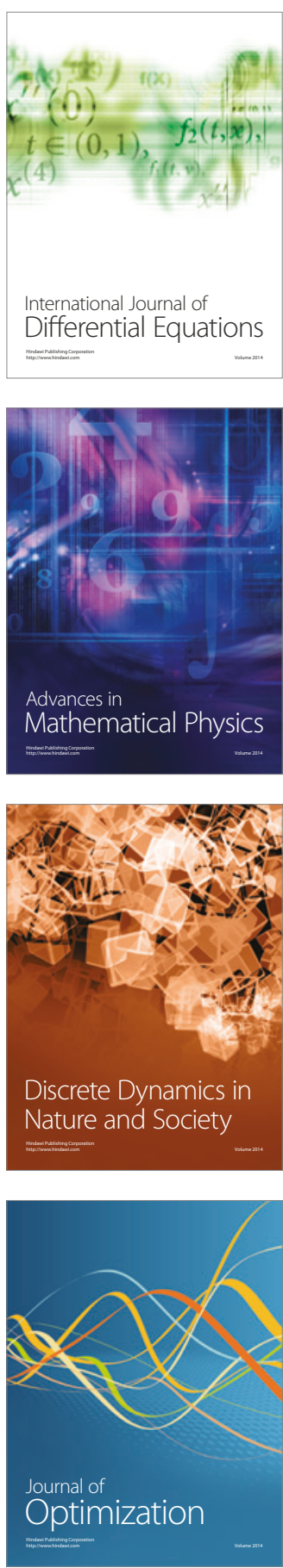